\section{REVISTA BRASILEIRA DE QUALIDADE DE VIDA}

\title{
Estilo de vida e prática de atividade física em colaboradores paranaenses
}

\author{
Lifestyle and physical activity in workers of the Paraná State
}

\author{
Neiva Leite \\ Universidade Federal do Paraná - UFPR - Curitiba - Brasil \\ neivaleite@gmail.com \\ Fabrício Cieslak \\ Universidade Federal do Paraná - UFPR - Curitiba - Brasil \\ facieslak@gmail.com \\ Ana Claudia Vecchi Osiecki \\ Universidade Federal do Paraná - UFPR - Curitiba - Brasil \\ a-na2@hotmail.com \\ Juan Alexandre Bizinelli \\ Universidade Federal do Paraná - UFPR - Curitiba - Brasil \\ juanbizi@hotmail.com \\ Luciana da Silva Timossi \\ Universidade Federal do Paraná - UFPR - Curitiba - Brasil \\ $\mathrm{CNPq}$ - Bolsista de Pesquisa \\ lucianatimossi@yahoo.com.br \\ Guanis de Barros Vilela Junior \\ Universidade Estadual de Ponta Grossa - UEPG - Ponta Grossa - Brasil \\ Universidade Metodista de Piracicaba - UNIMEP - Piracicaba - Brasil \\ guanis@gmail.com
} Universidade Tecnológica Federal do Paraná - UTFPR - Ponta Grossa - Brasil

\section{Resumo}

O objetivo foi determinar os fatores de estilo de vida associados à prática de atividade física (AF) de colaboradores do estado do Paraná. Participaram 907 indivíduos (646 homens e 261 mulheres), selecionados de forma não-aleatória. Utilizou-se o questionário QVS-80 para avaliação de dados sócio-demográficos, hábitos de AF, fumo e consumo de álcool. Analisaram-se as diferenças de proporções pelo teste Qui-quadrado, considerando significativas $\mathrm{p} \leq 0,05$. O índice de sedentarismo foi maior entre as mulheres $(78 \%)$ do que nos homens $(64 \% ; \mathrm{p}<0,001)$. As mulheres com maiores níveis de escolaridade apresentaram maior proporção de prática de $\operatorname{AF}(\mathrm{p}<0,05)$. Os homens não apresentaram associação entre as variáveis sócio-demográficas e AF semanal. Houve diferença significativa entre os gêneros em relação à preferência por modalidades de $\mathrm{AF}(\mathrm{p}=0,001)$ e também aos motivos da realização das mesmas, destacando-se motivos médicos e estéticos $(\mathrm{p}<0,01)$. Nos homens, o tabagismo ocorreu em $17 \%$ e o consumo de álcool em $8 \%$ no total. Nos praticantes de $\mathrm{AF}$, o tabagismo (15\%) foi associado a menores níveis de instrução e renda familiar ( $\mathrm{p}<0,005)$. Nas mulheres, o tabagismo ocorreu em 8\% das colaboradoras. Nas praticantes de AF, o tabagismo ocorreu em semelhante proporção (8\%). O consumo de álcool não teve associação com variáveis socio-demográficas para ambos os gêneros. Conclui-se que os colaboradores pesquisados apresentaram elevadas prevalências de sedentarismo, e valores dentro dos níveis já pesquisados 
anteriormente para tabagismo e consumo de álcool. Quanto à prática de $\mathrm{AF}$, nos homens não houve associação com as variáveis sócio-demográficas e nas mulheres se associou a menor faixa etária e ao maior nível de instrução.

Palavras-chave: Atividade física; idade, escolaridade, fatores sócio-econômicos, trabalhadores.

\begin{abstract}
The purpose of this paper was to determine what lifestyle factors are correlated to the practice of physical activities (PA) through the observation of the participants' habits. The subjects under analysis were 907 individuals from the state of Paraná (646 men and 261 women) selected in a norandom way. The QVS-80 questionnaire was used to evaluate socio-demographic data besides PA, smoking and alcohol consumption habits. The differences in proportion were analyzed using the test Qui-square, considering the $\mathrm{p}$-value $\mathrm{p} \leq 0,05$ significant. The sedentary lifestyle rates were higher among women $(78 \%)$ than among men $(64 \% ; \mathrm{p}<0,001)$. Women with higher education levels presented larger proportion of PA practice $(\mathrm{p}<0,05)$. Men haven't presented any association between the two variables socio-demographic and weekly PA practice. There was a significant difference between genders concerning their modalities of PA practice preferences $(p=0,001)$ and it was also meaningful the divergence in the reasons which motivated them to practice them. Medical and a esthetic $(\mathrm{p}<0,01)$ reasons prevailed in women motivation. Regarding men tabagism occurred in $17 \%$ and alcohol consumption in $8 \%$ of the total cases. In practitioners of PA group, smoking $(15 \%)$ was associated with lower levels of education and family income $(\mathrm{p}<0.005)$. In women, smoking occurred in $8 \%$ of the collaborators. In the group of PA adepts, smoking occurred in a similar proportion (8\%). Alcohol consumption was not associated with socio-demographic variables, in both genders. It is concluded that the employees surveyed had high prevalence of sedentary lifestyle, but smoking and alcohol consumption were similar of others scientifics publications. As the practice of PA in men there was no association with sociodemographic variables and women was associated with lower age and higher level of education.
\end{abstract}

Keywords: Physical activity, age, education, socioeconomic factors, workers

\title{
1. Introdução
}

O estilo de vida da população modificou-se nas últimas décadas, acarretando um aumento do número de pessoas que se tornaram sedentárias, principalmente em países industrializados (PITANGA, 2005). As menores oportunidades de praticar atividades físicas se relacionam tanto a morbidade quanto a mortalidade. As doenças infecciosas foram substituídas por um grande aumento das enfermidades crônicas e daquelas causadas pelo homem (MURRAY; LOPEZ, 1997). Pesquisas têm mostrado associação entre os baixos níveis de atividade física e o desenvolvimento de doenças cardiovasculares, câncer, diabetes e agravos na saúde mental (PITANGA, 2005; MONTEIRO, 1996) e a diminuição da qualidade de vida da população (FONTES; VIANNA, 2009), fatores que afetam também os trabalhadores.

Esse atual estilo vida trouxe muitas preocupações que estimularam o conhecimento científico na área da saúde ocupacional. Estudos procuram buscar informações e conhecer os fatores de risco e proteção para enfermidades crônicas em trabalhadores (PINHEIRO, 2006). Tem-se comprovado a associação entre as doenças cardiovasculares e um conjunto de fatores de risco, tais como: tabagismo, consumo excessivo de álcool, excesso de peso, hipertensão arterial, hipercolesterolemia, baixo consumo de frutas e hortaliças e sedentarismo (OMS - ORGANIZAÇÃO MUNDIAL DA SAÚDE, 2003).

O desenvolvimento da tecnologia tem melhorado significativamente as condições de trabalho nas empresas. Entretanto, a comodidade ocasionada por esse desenvolvimento e tecnologia no trabalho e no lazer, facilitam demasiadamente a opção desenfreada de um estilo de vida caracterizado pela inatividade física e por uma alimentação inadequada. Isto resulta em um excesso de gordura corporal que é um dos problemas que está associado a esse estilo de vida moderno 
(SANTOS; COELHO, 2003) e que determina inúmeras doenças. Têm-se detectado nos últimos anos um aumento na prevalência da obesidade em todas as faixas etárias principalmente em países desenvolvidos (CAMPOS; GOMES; OLIVEIRA, 2008).

Há a imprescindível necessidade de conscientização e reconhecimento de que os hábitos de vida modificam o desenrolar da história evolutiva tanto da saúde como também do adoecer (JARDIM, 2007). Dentre alguns hábitos inadequados, está o uso de produtos derivados do tabaco, que é apontado mundialmente como fator causador de prejuízos à saúde e danos irreversíveis aos usuários (INCA, 2008). Desde 1993, o tabagismo é considerado dependência química devido ao uso de substâncias psicoativas (Classificação Internacional de doença - CID 10) da OMS. É considerada um dos maiores e principais fatores de risco para muitas outras doenças, inclusive levando ao óbito (BRASIL, 2003).

Outro fator nocivo à saúde é o álcool, considerado um psicotrópico, ou seja, atua sobre o cérebro, alterando o comportamento e desenvolvendo, inclusive, dependência em quem o consome regularmente (OLIVEIRA; LUIZ, 1996). O álcool é uma das poucas drogas que tem seu consumo admitido e até incentivado pela sociedade. Apesar de aceito nos meios sociais, o consumo quando em excesso, traz à tona sérios transtornos, tais como acidentes de trânsito ou violência associada à embriaguez (MOREIRA; BELMONTE, 2008), acidentes de trabalho em curto e médio prazo (MINISTÉRIO DA SAÚDE, 2004; AMARAL; MALBIERGER, 2004), e mesmo à dependência ao álcool, conhecido como alcoolismo (ou etilismo) em longo prazo (OLIVEIRA; LUIZ, 1996; MOREIRA; BELMONTE, 2008).

Este trabalho objetivou determinar os fatores de estilo de vida associados à prática de atividade física de trabalhadores do estado do Paraná.

\section{Metodologia}

A coleta de dados foi realizada entre Julho de 2007 e Julho de 2008, participando da pesquisa 1061 colaboradores de empresas conveniadas ao SESI (Serviço Social da Indústria) do estado do Paraná. A população foi composta por trabalhadores de ambos os sexos e que exerciam suas atividades laborais em diferentes turnos de trabalho (manhã, tarde ou noite). A seleção dos participantes foi realizada de forma não-aleatória e por acessibilidade, sendo que, o número de colaboradores analisados em cada empresa e região foi baseado no cálculo amostral da mesma, para uma prevalência de 50\%, intervalo de confiança de $95 \%$ e erro amostral máximo de $3 \%$. Para o cálculo amostral foi utilizada equação a seguir:

$$
\begin{aligned}
& \text { Equação 1 - Equação para estimar o tamanho da amostra em populações finitas } \\
& \qquad \begin{array}{c}
n=\frac{N \sigma^{2}\left(z_{\alpha / 2}\right)^{2}}{(N-1) E^{2}+\sigma^{2}\left(z_{\alpha / 2}\right)^{2}} \\
\text { Fonte: Triola (2005) }
\end{array}
\end{aligned}
$$

Onde $\mathrm{n}=$ Tamanho amostra; $\mathrm{N}=$ Tamanho da população; $\sigma=$ Desvio padrão populacional; $E=$ Margem de erro da estimativa de um parâmetro populacional; $Z_{\alpha / 2}=$ Valor crítico, relacionado com o grau de confiança adotado, da distribuição normal padronizada.

Os critérios de exclusão da amostra foram determinados a partir do preenchimento incorreto e/ou ilegível dos questionários. A partir destes critérios, 154 questionários foram excluídos do resultado da pesquisa, reduzindo a amostra para 907 colaboradores (646 homens e 261 mulheres). O estudo foi submetido ao comitê de ética do Setor da Saúde da Universidade Federal do Paraná, atendendo a resolução 196/96 sob o seguinte registro: CEP/SD: 622.159.08.09; CAAE 31.84.0.000.091-08.

A coleta de dados foi realizada a partir da utilização do questionário o QVS-80, de avaliação da qualidade de vida e saúde de trabalhadores com 80 perguntas objetivas (LEITE et al., 2008). Consideraram-se as respostas das questões de números 29 a 37.

$\mathrm{Na}$ avaliação das questões sócio-demográficas utilizaram-se os seguintes critérios: 
- Nível de atividade física: considerou-se como indivíduo ativo aquele que atingiu no mínimo 150 minutos de atividade física por semana segundo recomendação da ACSM (2010), conforme o total das respostas nas questões $n^{\circ} 32$ a 37 . Avaliou-se em cada questão o valor médio em minutos. Por exemplo, se o relatado foi de 30 minutos a 60 minutos de atividade, considerou-se 45 minutos para o cálculo da pratica de atividade física. Ao final somaram-se todos os minutos relatados em diferentes atividades.

- Tabagismo: o hábito de tabagismo foi avaliado pela utilização atual ou não de tabaco, conforme respostas obtidas na questão $\mathrm{n}^{\circ} 29$. Foram considerados como não fumantes todos os indivíduos que nunca fumaram e também aqueles com histórico passado de fumo (ex-fumantes).

- Consumo de álcool: o hábito do consumo de bebida alcoólica foi classificado como "adequado" e "inadequado", conforme a análise da questão no 30. Considerou-se como "adequado" quando o indivíduo não bebe ou consome de 1 a 4 doses semanalmente. Denominou-se como "inadequado" ou consumo habitual, quando referido o consumo de 5 até 14 doses ou mais semanalmente. Esta linha de corte foi estabelecida de acordo com o estudo de Niroomand et al. (2004).

Para a análise dos resultados os dados sócio-demográficos como sexo, idade, escolaridade, estado civil, renda familiar foram cruzados através do teste Qui-quadrado com dados a respeito da atividade física, hábitos de fumo e consumo de álcool, considerando significativos valores menores que $5 \%(\mathrm{p} \leq 0,05)$.

\section{Resultados e Discussão}

\subsection{Prática de atividade física}

A prevalência da inatividade física foi uma característica muito presente nos colaboradores deste estudo, atingindo $78 \%$ das mulheres e $64 \%$ dos homens, em que apenas $31 \%$ dos indivíduos analisados praticavam atividades físicas freqüentemente ou muito frequentemente. $\mathrm{O}$ índice de prática de exercícios físicos foi menor entre as mulheres $(22 \%)$ em relação aos homens (36\%; $\mathrm{p}<0,001)$. Este resultado mostra-se superior aos índices nacionais divulgados pelo Ministério da Saúde (2008), em que apenas $16,4 \%$ da população são considerados ativos de acordo com as recomendações internacionais, mesmo considerando o ligeiro aumento em relação a $2007(15,5 \%)$ e a 2006 (14,9\%). Os resultados estão de acordo aos observados por Sávio et al. (2008), em que os colaboradores do distrito federal apresentaram $20 \%$ de prática regular de atividade física ou esporte, com diferenças significativas entre os índices masculinos $(27,3 \%)$ e femininos $(13,1 \%)$.

A associação das variáveis sócio-demográficas com a prática da recomendação mínima de atividade física semanal não se mostrou significativa entre os homens. As características sóciodemográficas dos homens podem ser observadas na Tabela 1.

$\mathrm{Na}$ presente pesquisa não houve diferenças entre a prática de atividade física segundo as faixas de idade, escolaridade e renda familiar no gênero masculino. No entanto, verificou-se maior tendência a prática freqüente de atividade física em homens jovens, com idade abaixo de 30 anos com maior escolaridade, ensino superior completo ou pós-graduação e com maior renda familiar per capita, em que $50 \%$ dos praticantes de atividade física regular possuem rendimento acima de 10 salários mínimos. Estes resultados estão de acordo com o padrão de gasto energético e a padrão alimentar citados por Mendonça e Anjos (2004) os quais evidenciam que o aumento do sobrepeso/obesidade, atualmente, é mais intenso entre as classes menos favorecidas, segmento da população em que o acesso a alimentos não é contínuo e nem é caracterizado como uma população que pratica atividades físicas com um padrão regular. 
Tabela 1 - Distribuição percentual da prática de atividade física segundo características sócio-demográficas

\begin{tabular}{|c|c|c|c|c|c|}
\hline \multirow{2}{*}{$\frac{\text { Variáveis de Estudo }}{\text { Idade (em anos) }}$} & \multicolumn{4}{|c|}{ Prática de Atividade Física * } & \multirow[t]{2}{*}{ p-valor } \\
\hline & Sim & $\%$ & Não & $\%$ & \\
\hline $16-30$ & 127 & $41 \%$ & 185 & $59 \%$ & 0,36 \\
\hline $31-40$ & 58 & $32 \%$ & 125 & $68 \%$ & \\
\hline $41-50$ & 25 & $25 \%$ & 75 & $75 \%$ & \\
\hline$>51$ & 10 & $23 \%$ & 33 & $77 \%$ & \\
\hline \multicolumn{6}{|l|}{ Escolaridade } \\
\hline Ensino fundamental & 90 & $34 \%$ & 177 & $66 \%$ & 0,885 \\
\hline Ensino médio & 91 & $35 \%$ & 172 & $65 \%$ & \\
\hline Ensino superior & 40 & $36 \%$ & 70 & $64 \%$ & \\
\hline Renda Familiar (em reais) & & & & & \\
\hline de 207,00 até 927,00 & 101 & $35 \%$ & 184 & $65 \%$ & 0,36 \\
\hline de 928,00 até $4.648,00$ & 99 & $31 \%$ & 216 & $69 \%$ & \\
\hline Acima de 4.649,00 & 8 & $44 \%$ & 10 & $56 \%$ & \\
\hline
\end{tabular}

As variáveis sócio-demográficas femininas mostraram-se diferenciados de acordo com os resultados demonstrados na tabela 2 .

Tabela 2 - Distribuição percentual de prática de atividade física segundo características sócio-demográficas entre mulheres

\begin{tabular}{|c|c|c|c|c|c|}
\hline \multirow{2}{*}{$\begin{array}{c}\text { Variáveis de Estudo } \\
\text { Idade (em anos) }\end{array}$} & \multicolumn{4}{|c|}{ Prática de Atividade Física * } & \multirow[t]{2}{*}{ p-valor ** } \\
\hline & Sim & $\%$ & Não & $\%$ & \\
\hline $16-30$ & 39 & $28 \%$ & 101 & $72 \%$ & 0,006 \\
\hline $31-40$ & 15 & $21 \%$ & 58 & $79 \%$ & \\
\hline $41-50$ & 4 & $11 \%$ & 31 & $89 \%$ & \\
\hline$>51$ & 0 & $0 \%$ & 8 & $100 \%$ & \\
\hline \multicolumn{6}{|l|}{ Escolaridade } \\
\hline Ensino fundamental & 15 & $19 \%$ & 65 & $81 \%$ & 0,433 \\
\hline Ensino médio & 25 & $22 \%$ & 89 & $78 \%$ & \\
\hline Ensino superior & 18 & $28 \%$ & 47 & $72 \%$ & \\
\hline \multicolumn{6}{|l|}{ Renda Familiar (em reais) } \\
\hline de 207,00 até 927,00 & 22 & $21 \%$ & 81 & $79 \%$ & 0,096 \\
\hline de 928,00 até $4.648,00$ & 25 & $21 \%$ & 96 & $79 \%$ & \\
\hline Acima de 4.649,00 & 5 & $50 \%$ & 5 & $50 \%$ & \\
\hline
\end{tabular}

Observou-se uma tendência da maior prática de AF entre as mulheres mais jovens com idade menores ou iguais a 30 anos, com maior renda familiar per capita (classe A) e com maior escolaridade $(\mathrm{p}<0,05)$. O maior índice de sedentarismo feminino encontra-se na faixa etária acima dos 51 anos, em que $77 \%$ não atingiram a recomendação mínima de 30 minutos diários de atividades físicas moderadas. Segundo Sávio (2008), existe relatos controversos na literatura quanto ao efeito da idade nos níveis de sedentarismo da população. Porém estudos da OMS relatam diminuição na prática da atividade física com o aumento da idade (OMS, 2004).

A recomendação mínima de 30 minutos diários de atividade física moderada foi considerada como prática regular de atividades físicas nesta pesquisa. Esses valores foram estabelecidos para a população americana adulta (PATE et al., 1995), na perspectiva de que tal prática acarretasse 
dispêndio de $1.000 \mathrm{kcal}$ por semana, ou 90 minutos por semana, quantidades recomendadas para reduzir substancialmente a taxa de mortalidade (BLAIR et al., 1992, 1995).

Porém dados mais recentes como do National Institute of Health (2000) mostram que o nível de $1.000 \mathrm{kcal}$ por semana é atualmente reconhecido como muito pequeno para a manutenção de estilo de vida saudável, e, possivelmente para a redução do risco relativo de mortalidade por doenças cardiovasculares (WILLIAMS, 2001). A recomendação do colégio americano de medicina esportiva para promover a saúde e a perda de peso é o gasto semanal em atividades físicas iguais ou superiores a $2000 \mathrm{kcal}$ ou 150 a 200 minutos por semana (ACSM, 2010).

$\mathrm{Na}$ tabela 3, verificam-se as preferências das modalidades de atividades físicas dos colaboradores paranaenses quanto ao gênero. Notou-se que a caminhada foi a modalidade mais referida entre homens e mulheres, entre as atividades físicas praticadas semanalmente e que atendem a recomendação mínima de 30 minutos diários ou 150 minutos por semana. A segunda atividade mais realizada pelos homens foram as esportivas como futebol, vôlei, basquete e futebol de salão. A preferência feminina configurou-se na musculação. Os dados obtidos neste estudo mostraram diferenças nas preferências masculinas e femininas na escolha das modalidades de atividades físicas ( $\mathrm{p}=0,001)$. Em concordância com estes resultados, Salles et al. (2003) observaram que a participação masculina é maior entre atividades físicas coletivas ( futebol, voleibol e tênis) e entre as mulheres, as atividades preferidas foram a caminhada, ginástica e dança.

Tabela 3 - Modalidades de atividades físicas praticadas pelos colaboradores

\begin{tabular}{lccccc}
\hline \multicolumn{1}{c}{ Variáveis de Estudo } & \multicolumn{2}{c}{ Homens } & Mulheres & p-valor ** \\
\hline & $\mathbf{N}$ & $\mathbf{\%}$ & $\mathbf{N}$ & $\%$ & \\
Caminhada & 149 & $23 \%$ & 28 & $11 \%$ & $\mathrm{p}=0,001$ \\
Corrida & 77 & $12 \%$ & 3 & $1 \%$ & \\
Musculação & 41 & $7 \%$ & 13 & $6 \%$ & \\
Atividades Aquáticas & 13 & $2 \%$ & 2 & $0 \%$ & \\
Esporte & 95 & $14 \%$ & 5 & $2 \%$ & \\
Dança/Ginástica & 29 & $4 \%$ & 6 & $3 \%$ & \\
** Teste Qui-quadrado & & & & & \\
Fonte: Pesquisa de campo (2008) & & & & &
\end{tabular}

Em relação aos motivos da prática de atividade física, os colaboradores masculinos as realizavam principalmente por motivos médicos (78\%), seguido por motivos estéticos (67\%). Enquanto que nas mulheres, a prática estava relacionada a estética (63\%), seguido também por motivos médicos $(60 \%)$. Os resultados quanto aos motivos de práticas por gênero no total dos colaboradores estão descritos na tabela 4.

Tabela 4 - Distribuição percentual dos motivos da prática de atividade física de lazer entre os gêneros

\begin{tabular}{lcccccccc}
\hline \begin{tabular}{l} 
Motivo relatado da prática \\
\multicolumn{1}{c}{ de atividade física }
\end{tabular} & \multicolumn{2}{c}{ Homens } & & & \multicolumn{2}{c}{ Mulheres } \\
\hline & Sim & \% & Não & \% & Sim & \% & Não & \% \\
Prazer da Atividade & 260 & $41 \%$ & 372 & $59 \%$ & 64 & $26 \%$ & 185 & $74 \%$ \\
Relações sociais & 181 & $29 \%$ & 450 & $71 \%$ & 35 & $15 \%$ & 206 & $85 \%$ \\
Médicos & 499 & $78 \%$ & 138 & $22 \%$ & 187 & $60 \%$ & 127 & $40 \%$ \\
Condicionamento físico & 224 & $35 \%$ & 410 & $65 \%$ & 57 & $23 \%$ & 190 & $77 \%$ \\
Estético & 425 & $67 \%$ & 211 & $33 \%$ & 156 & $63 \%$ & 91 & $37 \%$ \\
Fonte: Pesquisa de campo (2008) & & & & & & & \\
\hline
\end{tabular}

* Atinge recomendação mínima de 30 minutos diários

** Teste Qui-quadrado

$\mathrm{O}$ motivo estético como fator à prática de atividade física merece destaque, porque atingiu percentual de 67\% nos homens e 63\% nas mulheres. Salles Costa et al. (2003) afirmaram que a prática de atividades físicas no universo feminino ganha grande proporção por meio da busca do corpo delineado e controle do peso corporal, fenômeno observado, sobretudo, em sociedades mais desenvolvidas. Para Goldenberg (2002), o comportamento das mulheres em busca de um corpo 
esguio, definido e perfeito, reflete nada mais que a vulnerabilidade e à influência da cultura da estética na nossa sociedade. Porém, no presente estudo, a influência e valorização da estética também foram verificadas nos colaboradores masculinos ao considerar os motivos mais freqüentes à prática de atividade física.

Estes resultados podem ser importantes indicadores para que as empresas possam melhor informar, planejar e direcionar as atividades aos seus colaboradores, garantindo assim uma possibilidade de maior adesão e incentivo à prática de atividades físicas regulares. Para LimongiFrança (2004), as ações promovidas pelas empresas e possíveis para manter as pessoas saudáveis, podem ser classificadas em ações de recuperação, proteção e promoção da saúde. As ações de recuperação da saúde são desenvolvidas com pessoas já doentes, para recuperá-las tanto do ponto de vista físico como do psicológico e do social. Enquanto as ações de proteção da saúde visam evitar a exposição dos indivíduos a agentes nocivos identificados, por exemplo, o emprego de equipamentos de proteção individual no trabalho e vacinação. Por último, as ações de promoção da saúde são mais abrangentes e extrapolam a preocupação de evitar doenças. Ter saúde ou ser saudável não significa apenas não estar doente, envolve satisfação, plenitude, integração social, atividade física, alimentação, exercício da cidadania e outros.

\subsection{Hábitos relacionados ao tabagismo}

Os fatores de risco e proteção para doenças crônicas no Brasil são avaliados regularmente por inquérito de vigilância telefônica (VIGITEL). No último ano, constatou-se uma freqüência de indivíduos fumantes de $15,2 \%$, ocorrendo $19,1 \%$ no sexo masculino e $11,9 \%$ no feminino, após estudo em 27 cidades brasileiras. A prevalência do hábito de fumar foi particularmente elevada entre homens $(24,6 \%)$ e mulheres $(14,7 \%)$ com até oito anos de escolaridade, excedendo em quase duas vezes o percentual observado em indivíduos com maior escolaridade (BRASIL, 2008).

Neste estudo, as prevalências de tabagismo ocorreram em $15 \%$ do total dos participantes (646 homens e 261 mulheres), sendo que em 18\% dos homens e 8\% das mulheres. Estes valores se mostraram abaixo dos valores médios da capital paranaense, considerando as prevalências totais e para os gêneros divulgados pelo Ministério da Saúde (2009) e semelhantes ao VIGITEL (2008). No Brasil, as maiores frequiências de tabagismo se encontram na região Sul, mais especificamente em Porto Alegre $(25,2 \%)$, Curitiba $(21,8 \%)$ e Florianópolis $(21,4 \%)$. Quanto ao gênero, no grupo masculino, Porto Alegre $(28,2 \%)$ lidera a lista dos maiores fumantes, seguido por Belo Horizonte (26,1\%), Florianópolis $(24,5 \%)$ e Curitiba $(24,3 \%)$. Entre as mulheres, as maiores taxas ocorreram em Porto Alegre $(22,9 \%)$, Curitiba $(19,7 \%)$ e Florianópolis $(18,9 \%)$ (MINISTÉRIO DA SAÚDE, 2009).

$\mathrm{Na}$ avaliação desse hábito em colaboradores praticantes de atividades físicas ( $\mathrm{n}=309$ homens; $n=65$ mulheres), detectou-se que $17 \%$ dos homens $(n=52)$ e $8 \%$ das mulheres $(n=5)$ utilizavam tabaco, sendo a grande maioria, pessoas na faixa dos 16 aos 30 anos. As prevalências de tabagismo encontradas em praticantes de atividades físicas foram mais baixas do que na população curitibana. Ao avaliar a prevalência de tabagismo em não praticantes de atividades físicas, mesmo sem grande significância, encontrou-se $18 \%$ entre os homens (62 indivíduos: $p=0,554$ ) e $8 \%$ nas mulheres ( 15 individuos: $\mathrm{p}=0,907$ ), valores de consumo de tabaco semelhantes aos praticantes de atividades físicas.

Nesta pesquisa, focalizaram-se os homens praticantes de atividades físicas, tabagistas ou não, relacionando ao nível de escolaridade, renda e faixa etária. Houve associação do tabagismo e grau de instrução ( $\mathrm{p}=0,006)$, em que indivíduos com maior grau de instrução apresentam menor nível de consumo de tabaco; já o consumo do tabaco, associado à renda familiar, apontou que, quanto maior a renda, menor o consumo de tabaco $(\mathrm{p}=0,014)$. Finalmente, associando o consumo tabagista com faixa etária, foi possível perceber também que as idades acima de 40 anos apresentaram maior consumo (0,043), havendo significância neste e nos demais aspectos relacionados anteriormente (Tabela 5). 
Tabela 5 - Distribuição percentual do Tabagismo segundo características sócio-demográficas entre homens praticantes de atividades físicas

\begin{tabular}{|c|c|c|c|c|c|}
\hline \multirow{2}{*}{$\begin{array}{c}\text { Variáveis de Estudo } \\
\text { Idade (em anos) }\end{array}$} & \multicolumn{4}{|c|}{ Consumo de Tabaco } & \multirow[t]{2}{*}{ p-valor } \\
\hline & Sim & $\%$ & Não & $\%$ & \\
\hline $16-30$ & 28 & $17 \%$ & 140 & $83 \%$ & $* * 0,043$ \\
\hline $31-40$ & 9 & $11 \%$ & 72 & $89 \%$ & \\
\hline $41-50$ & 13 & $31 \%$ & 29 & $69 \%$ & \\
\hline$>51$ & 2 & $12 \%$ & 15 & $88 \%$ & \\
\hline \multicolumn{6}{|l|}{ Escolaridade } \\
\hline Ensino fundamental & 32 & $24 \%$ & 101 & $76 \%$ & $* * 0,006$ \\
\hline Ensino médio & 17 & $14 \%$ & 104 & $86 \%$ & \\
\hline Ensino superior & 3 & $6 \%$ & 50 & $94 \%$ & \\
\hline \multicolumn{6}{|l|}{ Renda Familiar (em reais) } \\
\hline de 207,00 até 927,00 & 33 & $24 \%$ & 107 & $76 \%$ & $* * 0,014$ \\
\hline de 928,00 até $4.648,00$ & 18 & $13 \%$ & 125 & $87 \%$ & \\
\hline Acima de $4.649,00$ & 0 & 0 & 12 & $100 \%$ & \\
\hline
\end{tabular}

** Teste Qui-quadrado

Fonte: Pesquisa de campo (2008)

A OMS (2003) estima que há em torno de 1 bilhão e 300 mil fumantes em todo o mundo e 2 bilhões de fumantes passivos, sendo que 700 milhões são crianças, mostrando que metade da população terrestre está exposta direta ou indiretamente aos efeitos nocivos do cigarro. Hoje, o tabaco mata 1 em cada 10 pessoas. Estima-se que se não houver uma tendência à queda destes números, em 2020 ocorrerão 10 milhões de mortes pelo fumo (ROSEMBERG, 2003).

A prevalência de tabagismo foi semelhante entre colaboradores praticantes e não praticantes de atividades físicas nesta pesquisa, evidenciando que o consumo de cigarros está associado a outras variáveis como: falta de informação e o "status" social do homem em faixas de menor renda familiar. Segundo o INCA (2008) em 2020, de cada 10 mortes devido ao fumo, 7 ocorrerão em países em desenvolvimento, devido à insuficiência de informações sobre os malefícios do tabaco, além disto, 100 mil jovens começam a fumar a cada dia. Em muitos países existe uma correlação entre tabagismo, baixa renda e baixo nível de escolaridade. Na China, indivíduos sem escolaridade têm uma probabilidade cerca de sete vezes maior de serem fumantes do que indivíduos com ensino superior. No Brasil, indivíduos com baixo nível de escolaridade tem probabilidade cinco vezes maior (WORLD BANK, 2003). A (Tabela 6) apresenta os hábitos de tabagismo nas colaboradoras.

Tabela 6 - Distribuição percentual do Tabagismo segundo características sócio-demográficas entre mulheres praticantes de atividades físicas

\begin{tabular}{|c|c|c|c|c|c|}
\hline Variáveis de Estudo & \multicolumn{4}{|c|}{ Consumo de Tabaco } & p-valor \\
\hline Idade (em anos) & Sim & $\%$ & Não & $\%$ & \\
\hline $16-30$ & 3 & $8 \%$ & 34 & $92 \%$ & 0,768 \\
\hline $31-40$ & 2 & $11 \%$ & 16 & $89 \%$ & \\
\hline $41-50$ & 0 & 0 & 6 & 100 & \\
\hline$>51$ & 0 & 0 & 4 & 100 & \\
\hline \multicolumn{6}{|l|}{ Escolaridade } \\
\hline Ensino fundamental & 0 & 0 & 12 & $100 \%$ & 0,538 \\
\hline Ensino médio & 3 & $9 \%$ & 30 & $91 \%$ & \\
\hline Ensino superior & 2 & $10 \%$ & 18 & $90 \%$ & \\
\hline \multicolumn{6}{|l|}{ Renda Familiar (em reais) } \\
\hline de 207,00 até 927,00 & 1 & $5 \%$ & 21 & $95 \%$ & 0,677 \\
\hline de 928,00 até $4.648,00$ & 3 & $9 \%$ & 31 & $91 \%$ & \\
\hline Acima de 4.649,00 & 0 & 0 & 5 & 0 & \\
\hline
\end{tabular}


Quanto aos hábitos de tabagismo nas colaboradoras praticantes de atividades físicas (Tabela 6), percebe-se um baixo índice de indivíduos fumantes deste sexo, mostrando não ser significativo nenhuma destas associações com o hábito do tabagismo, seja entre idade e tabagismo $(\mathrm{p}=0,768)$, escolaridade e tabagismo $(\mathrm{p}=0,538)$ ou mesmo renda familiar e consumo de tabaco $(\mathrm{p}=0,677)$.

Se por um lado, tais dados relacionados ao sexo feminino não se mostraram significativos, em outro panorama, os percentuais encontrados coincidem com os valores já anteriormente descritos em pesquisas anteriores, ou seja, o hábito do tabagismo tem percentuais maiores dentre os homens do que entre as mulheres, principalmente em menores níveis de escolaridade (MINISTÉRIO DA SAÚDE, 2009).

\subsection{Hábitos relacionados ao consumo de álcool}

Nesta pesquisa, as prevalências de consumo habitual de álcool ocorreram em $7 \%$ do total dos participantes (646 homens e 261 mulheres), sendo que em 8\% dos homens e 3\% das mulheres. Estes valores se mostraram superiores aos valores médios da capital paranaense, considerando as prevalências totais e para os gêneros divulgados pelo Ministério da Saúde (2009). Dentre as capitais com menores taxas, no todo, segue a cidade de Curitiba (4,6\%), Florianópolis $(4,7 \%)$ e São Paulo $(4,9 \%)$. Com relação ao gênero, no grupo masculino, as menores prevalências foram encontradas para a cidade de Florianópolis $(5,4 \%)$, seguido por São Paulo $(8,1 \%)$ e Curitiba $(8,3 \%)$, enquanto no grupo feminino, a cidade de Curitiba lidera novamente com $1,7 \%$, seguido por João Pessoa $(2,0 \%)$ e São Paulo (2,4\%) (MINISTÉRIO DA SAÚDE, 2009).

A dependência de álcool acomete atualmente de $10 \%$ a $12 \%$ da população mundial (OMS, 2004) e 11,2\% dos brasileiros entre 12 e 65 anos que vivem nas 107 maiores cidades do país (CARLINI et al., 2002). Num âmbito nacional, estudos passados demonstram que a análise com amostras de população geral eram poucos, devido à existência de agravantes, tais como diversidade geográfica, socioeconômica e cultural, mas encontraram prevalência para o alcoolismo entre 3 e $6 \%$ (SANTANA; ALMEIDA FILHO, 1987).

Outro estudo abrangendo Distrito Federal e 17 capitais brasileiras mostra a prevalência de consumo excessivo alcoólico um total de 8,4\%, sendo 13,26\% para homens e 4,67\% para mulheres, em nível nacional; este estudo também mostra que a prevalência do consumo excessivo do álcool no Brasil encontra seus maiores patamares na região Nordeste, mais especificamente em São Luís $(12,7 \%)$, em Recife $(12,2 \%)$ e em Fortaleza $(11,1 \%)$, além de valores significativos em Vitória $(12,4 \%)$ e Belo Horizonte (10,9\%); quanto ao gênero, no grupo masculino, Recife tem os maiores níveis, com 21,6\%, seguido por Fortaleza $(18,4 \%)$, São Luís $(17,8 \%)$ e Vitória $(17,3 \%)$; Entre as mulheres, lideram com maiores taxas o grupo de São Luís, com $8,9 \%$, seguido por Vitória $(8,1 \%)$, Belo Horizonte $(7,3 \%)$ e Rio de Janeiro $(6,2 \%)$.

A relevância de avaliar a prevalência do uso inadequado do álcool entre os trabalhadores está na prevenção de acidentes de trabalho. Segundo dados da Organização Mundial de Saúde (OMS, 2003), os problemas relacionados ao uso de álcool são responsáveis por 54\% dos acidentes de trabalho com afastamento e por $40 \%$ dos acidentes com morte (ODO et al, 2000). Agravando mais ainda estes dados, a Organização Internacional do Trabalho (OIT) acrescenta que trabalhadores com problemas relacionados ao álcool costumam faltar 5 a 7 vezes mais ao trabalho (26 dias por ano, em média) do que os trabalhadores sem estes problemas (OIT, 1993).

A presente pesquisa também avaliou o consumo de álcool nos trabalhadores paranaenses em relação ao hábito de praticar atividades físicas regulares. Verificou-se que os colaboradores não praticantes de atividades físicas apresentaram prevalência de $6 \%$ no consumo habitual de álcool, sendo $8 \%$ nos homens e $3 \%$ nas mulheres. Entre os colaboradores praticantes de atividades físicas foi detectado que $8 \%$ dos colaboradores masculinos e $3 \%$ do feminino, faz uso de bebidas alcoólicas de forma inadequada. Os trabalhadores praticantes e não praticantes de atividades físicas apresentaram índices idênticos de consumo de álcool.

As associações entre o consumo ou não de álcool em colaboradores masculinos praticantes de atividades físicas estão na Tabela 7. Não houve associações entre o uso habitual de álcool e 
renda familiar $(\mathrm{p}=0,160)$, escolaridade $(\mathrm{p}=0,155)$ e faixa etária $(0,913)$. A variável idade também não se mostrou significativa ao relacionar-se com consumo etílico $(\mathrm{p}=0,913)$, apesar do consumo ser maior entre os mais jovens (58\% do total situa-se na faixa etária dos 16 aos 30 anos, regredindo esta porcentagem na medida que a faixa etária aumenta).

Tabela 7 - Distribuição do Etilismo segundo características sócio-demográficas entre homens praticantes de atividades físicas regulares

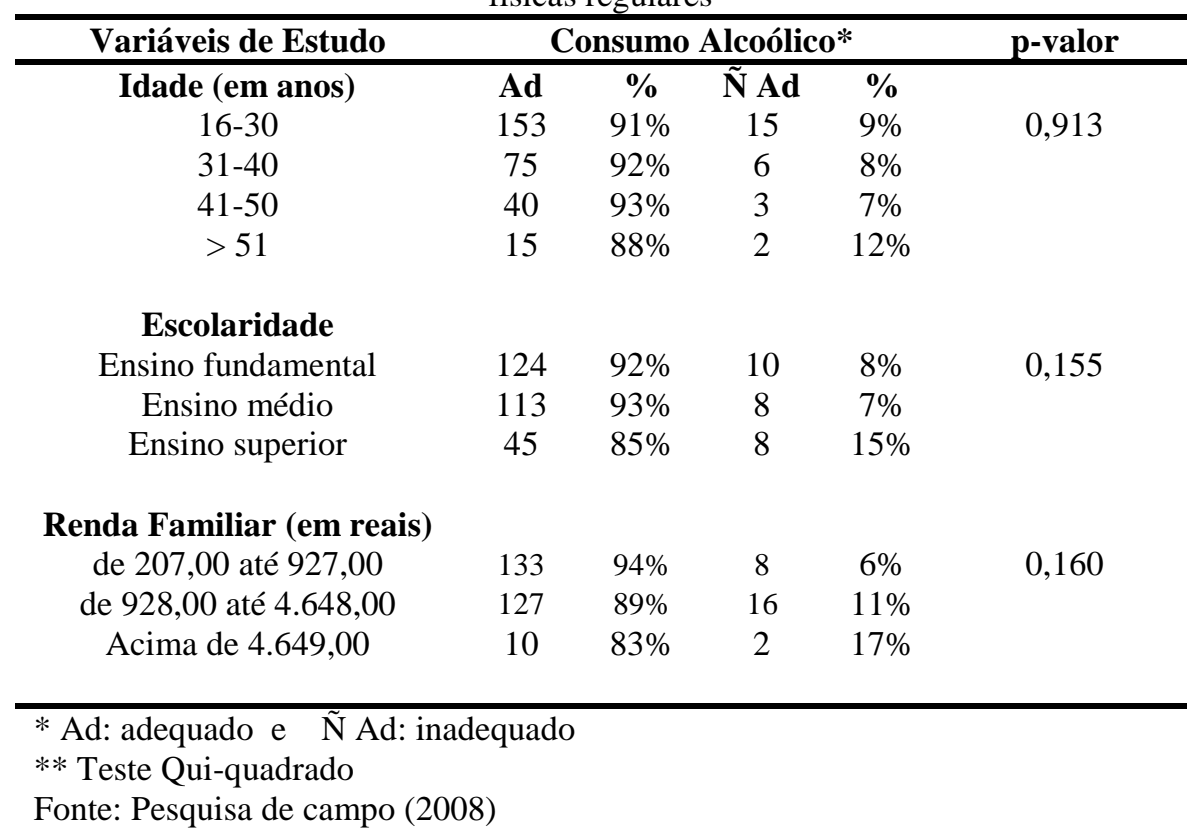

Tais dados demonstram que muitas características além do gênero, mas também etnia, idade, ocupação, grau de instrução e estado civil, podem influenciar o uso nocivo de álcool, bem como o desenvolvimento da dependência ao álcool (MARQUES; RIBEIRO, 2002). A prevalência de alcoolismo é maior entre os homens do que entre as mulheres, e o mesmo ocorrendo entre os mais jovens, especialmente na faixa etária dos 18 aos 24 anos, declinando com a idade (MARQUES; RIBEIRO, 2002; MINISTÉRIO DA SAÚDE, 2009).

A distribuição dos dados de consumo de álcool pela mulheres trabalhadoras praticantes de atividades físicas apresenta-se na Tabela 8.

Tabela 8 - Distribuição do Etilismo segundo características sócio-demográficas entre mulheres praticantes de atividades físicas regulares

\begin{tabular}{|c|c|c|c|c|c|}
\hline \multirow{2}{*}{$\begin{array}{c}\text { Variáveis de Estudo } \\
\text { Idade (em anos) }\end{array}$} & \multicolumn{4}{|c|}{ Consumo Alcoólico* } & \multirow[t]{2}{*}{ p-valor } \\
\hline & Ad & $\%$ & $\tilde{\mathbf{N}}$ Ad & $\%$ & \\
\hline $16-30$ & 36 & $97 \%$ & 1 & $3 \%$ & 0,872 \\
\hline $31-40$ & 17 & $94 \%$ & 1 & $6 \%$ & \\
\hline $41-50$ & 6 & $100 \%$ & 0 & 0 & \\
\hline$>51$ & 4 & $100 \%$ & 0 & 0 & \\
\hline \multicolumn{6}{|l|}{ Escolaridade } \\
\hline Ensino fundamental & 12 & $100 \%$ & 0 & 0 & 0,730 \\
\hline Ensino médio & 32 & $97 \%$ & 1 & $3 \%$ & \\
\hline Ensino superior & 19 & $95 \%$ & 1 & $5 \%$ & \\
\hline \multicolumn{6}{|l|}{ Renda Familiar (em reais) } \\
\hline de 207,00 até 927,00 & 20 & $91 \%$ & 2 & $9 \%$ & 0,160 \\
\hline de 928,00 até $4.648,00$ & 34 & $100 \%$ & 0 & 0 & \\
\hline Acima de 4.649,00 & 5 & $100 \%$ & 0 & 0 & \\
\hline
\end{tabular}


Ao tratar do consumo do álcool pelo gênero feminino praticante de atividades físicas, os dados não se mostraram significativos, assim como no gênero masculino, com nenhum dos aspectos cruzados, tendo valor de significância $\mathrm{p}=0,872$ para variável Idade, $\mathrm{p}=0,730$ para Escolaridade e $\mathrm{p}=0,160$ para variável Renda Familiar.

Em comparação ao público feminino não praticante de atividades físicas, nota-se que as prevalências não se mostraram significativas também, pois tanto para quem faz atividade como para quem não o faz, ambos os resultados afirmaram um valor de prevalência de consumo inadequado do álcool de 3\% (dentre as 65 praticantes, 02 têm ingestão desaconselhável, enquanto para as 187 não praticantes, 05 ingerem de forma inadequada, totalizando $3 \%$ para ambas as situações).

Através dos resultados anteriormente discutidos, é possível perceber que os valores para o gênero masculino e feminino, mesmo sem grande significância, refletem o panorama atual da capital do estado, já analisado em pesquisa anterior, ou seja, pautado em relatório do Ministério da Saúde (2009), a média alcançada quanto ao gênero masculino sendo de $8 \%$ contra $8,3 \%$ registrado na capital, e quanto ao gênero feminino sendo de $3 \%$ contra $1,7 \%$, tendo enfim, valores totais de $7 \%$ do público analisado como sendo usuário de álcool de forma desaconselhável.

Segundo dados do Mistério da Saúde, as doenças provocadas por fatores como sedentarismo, fumo, consumo excessivo de álcool e alimentação inadequada são as principais causas de morte no país (BRASIL, 2008). Em termos empresariais, o trabalhador que faz uso abusivo ou é dependente tem 3,6 vezes mais chances de causar acidentes no trabalho, 2,5 vezes mais chances de faltar, sem justificativas, 8 ou mais dias de trabalho, utiliza-se 3 vezes mais dos benefícios médicos, tem sua capacidade produtiva reduzida a $67 \%$, é punido disciplinarmente 7 vezes mais e é 5 vezes mais "queixoso" que trabalhadores não usuários (MINISTÉRIO DA SAÚDE, 2004). Conseqüentemente, as relações interpessoais são afetadas de tal forma que compromete o clima organizacional e a qualidade de vida destes trabalhadores.

Segundo especialistas, os indivíduos dependentes etílicos podem desenvolver várias doenças, dentre elas, com mais frequiência àquelas relacionadas ao fígado (esteatose hepática, hepatite alcoólica e cirrose), doenças do aparelho digestivo (gastrite, síndrome de má absorção e pancreatite) e do sistema cardiovascular (hipertensão e problemas cardíacos) (ROSA et al., 1998).

\section{Conclusão}

Neste estudo, os resultados indicaram que o índice de sedentarismo foi maior entre as mulheres do que nos homens. Houve maior tendência a prática freqüente de atividade física em jovens (idade $\leq 30$ anos), com maior escolaridade e com maior renda familiar per capita. Porém, os resultados quanto à associação destas variáveis sócio-demográficas com a prática da recomendação mínima de atividade física semanal se mostraram significativas somente para as mulheres. $\mathrm{O}$ hábito do tabagismo nos colaboradores masculinos mostrou-se associado aos menores grau de instrução e renda familiar e entre as mulheres não houve associação em relação a essas variáveis. $\mathrm{O}$ consumo de álcool não teve associação com a prática de atividades físicas e variáveis sócio-demográficas, para ambos os gêneros.

Conclui-se que os colaboradores pesquisados apresentaram elevadas prevalências de sedentarismo, e valores para tabagismo e consumo de álcool dentro dos níveis regionais divulgados pelo Ministério da Saúde. Sugerem-se novos estudos que avaliem a prevalência e a repercussão dos hábitos dos colaboradores sobre a qualidade de vida, qualidade de vida no trabalho e saúde ocupacional.

\section{Referências}

AMARAL, R.A., MALBIERGER, A. A avaliação de instrumento de detecção de problemas relacionados ao uso do álcool (CAGE) entre trabalhadores da Prefeitura do campus da Universidade de São Paulo (USP) - Campus capital. Revista Brasileira de Psiquiatria, São Paulo, v. 26, n. 3, p. 156-163, 2004. 
AMERICAN COLLEGE OF SPORTS MEDICINE; NEIL, F. G.; PESCATELLO, L. S.; THOMPSON, W. R. ACSM'S: Guidelines for exercise testing and prescription. 8. ed. Philadelphia: Wolters Kluwer, Lippincott Williams \& Wilkins, 2010.

BLAIR, S. N.; KOHL, H. W.; PAFFENBARGER, R. S.; CLARK, D. G.; COOPER, K. H.; GIBBONS, L. W. Physical fitness and all-cause mortality: a prospective study of healthy men and women. JAMA. v. 14, n. 273, p. 1093-1098, 1995.

BLAIR, S.N.; KOHL, H.W.; GORDON, N.F.; PAFFENBARGER, R.S. How much physical activity is good for health? Annual Review of Public Health, v. 13, p. 99-126, 1992.

BRASIL. Ministério da Saúde. Indicadores de morbidade e fatores de risco: IDB 2007. Brasília: Ministério da Saúde, 2007. Disponível em: <http://tabnet.datasus.gov.br/cgi/idb2007/d21a.htm>. Acesso em 03 junho de 2009.

BRASIL. Ministério da Saúde. Secretaria Executiva. Coordenação Nacional de DST/Aids. A Política do Ministério da Saúde para atenção integral a usuários de álcool e outras drogas. Brasília: Ministério da Saúde, 2003.

BRASIL. Ministério da Saúde. Secretaria de Políticas da Saúde. Saúde Brasil 2007. Brasília: Programa Nacional de promoção da Atividade Física. Brasília: Ministério da Saúde, 2008. Disponível em: <portal.saude.gov.br/portal/arquivos/pdf/coletiva_saude_061008.pdf>. Acesso em: 03 dezembro de. 2008.

BRASIL. Ministério da Saúde. Instituto Nacional do Câncer. Vigitel brasil 2008: Vigilância de fatores de risco e proteção para doenças crônicas por inquérito telefônico. Brasília: Ministério da Saúde, 2008. Disponível em <http://bvsms.saude.gov.br/bvs/publicacoes/vigitel_2008.pdf>. Acesso em 10 maio de 2009.

BRASIL. Ministério da Saúde, Instituto Nacional de Cãncer/Secretaria de Vigilância à Saúde. Inquérito Domiciliar sobre comportamentos de risco e mortalidade referida de doenças e agravos não-transmissíveis. Rio de Janeiro: Ministério da Saúde/INCA, 2004.

CAMPOS, L. F., GOMES, J. M. e OLIVEIRA, J. C. Obesidade Infantil, Actividade Física e Sedentarismo em crianças do $1^{\circ}$ ciclo do ensino básico da cidade de bragança (6 a 9 anos). Motri, v. 4, n. 3, p. 17-24, 2008.

CARLINI, E. A. et al. I Levantamento domiciliar sobre o uso de drogas psicotrópicas no Brasil: estudo envolvendo as 107 maiores cidades do país: 2001. São Paulo: UNIFESP Universidade Federal de São Paulo, 2002.

FONTES, A. C. D.; VIANNA, R. P. T. Prevalência e fatores associados ao baixo nível de atividade física entre estudantes universitários de uma universidade pública da região Nordeste - Brasil. Revista Brasileira de Epidemiologia, São Paulo, v. 12, n. 1, p. 20-29, 2009.

GOLDENBERG, M. (org.). Nu e Vestido: Dez Antropólogos Revelam a Cultura do Corpo Carioca. Rio de Janeiro: Record, 2002.

INSTITUTO NACIONAL DE CÂNCER. Coordenação de Prevenção e Vigilância. Brasil: advertências sanitárias nos produtos de tabaco 2009. Rio de Janeiro: INCA, 2008. Disponível em:

<http://www.inca.gov.br/tabagismo/publicacoes/brasil_advertencias_sanitarias_nos_produtos_de_ta baco2009b.pdf >. Acesso em: 29 maio de 2009. 
JARDIM, P. C. B. V.; et al . Hipertensão arterial e alguns fatores de risco em uma capital brasileira. Arquivos Brasileiros de Cardiologia, São Paulo, v. 88, n. 4, 2007.

LIMONGI-FRANÇA, A. C. Qualidade de Vida no Trabalho: QVT. 2. ed. São Paulo: Atlas, 2004.

MARQUES, A. C. P. R.; RIBEIRO M.; et al. Abuso e Dependência do Álcool. Associação Brasileira de Psiquiatria. $2002 . \quad$ Disponível em: <http://www.projetodiretrizes.org.br/projeto_diretrizes/002.pdf>. Acesso em: 29 maio de 2009.

MENDES, R. A.; LEITE, N. Ginástica laboral: princípios e aplicações práticas. São Paulo: Manole, 2008.

MENDONÇA, C. P.; ANJOS, L. A. Aspectos das práticas alimentares e da atividade física como determinantes do crescimento do sobrepeso/obesidade no Brasil. Cadernos de Saúde Pública, v. 20, n. 3, p. 698-709, 2004.

MONTEIRO, C. A. Velhos e novos males da saúde no Brasil: a evolução do país e de suas doenças. São Paulo: HUCITEC, 1996.

MOREIRA, T.C.; BELMONTE, E. L. et al. A violência comunitária e o abuso de álcool entre adolescentes: comparação entre sexos. Jornal de Pediatria, v. 84, n. 3, p. 244-250, 2008.

MURRAY, C. J. L.; LOPEZ, A. D. Mortality by cause for eight regions of the world: global burden of disease study. The Lancet. v. 349, n. 9061, p. 1269-1276, 1997.

NATIONAL INSTITUTES OF HEALTH. National American Association for the Study of Obesity. Obesity. The practical guide. Identification, evaluation, and treatment of overweight and obesity in adults. Bethesda: National Institutes of Health, 2000.

NIROOMAND, F. HAUER, O. TIEFENBACHER, C.P., KATUS, H.A. KUEBLER, W. Influence of alcohol consumption on restenosis rate after percutaneous transluminal coronary angioplasty and stent implantation. Heart. v. 90, n. 10, p. 1189 - 1193, 2004.

ODO, SA, ARAÚJO AC, SANTOS AF, TOLEDO FCP, YONAMINE M, SILVA OA, LEITE MC. Indicações e limites das análises toxicológicas para substâncias psicoativas. Revista de Psiquiatria Clínica. v. 27, n. 1, p. 50-56, 2000.

OLIVEIRA, E. R.; LUIS, M. A. V. Distúrbios relacionados ao álcool em um setor de urgências psiquiátricas. Caderno de Saúde Pública, v. 12, n. 2, p. 171-179, 1996.

ORGANIZAÇÃO INTERNACIONAL DO TRABALHO - INTERNATIONAL LABOUR OFFICE. Guiding principles adopted by the ILO Inter-regional Tripartite Experts Meeting on Drug and Alcohol Testing in the Workplace. Geneve, 1993.

ORGANIZAÇÃO MUNDIAL DA SAÚDE (World Health Organization (WHO). Global Status Report on Alcohol. Genebra: WHO, 2004. Disponível em: <http://whqlibdoc.who.int/publications/2004/9241562722_(425KB).pdf>. Acesso em: 10 maio 2009.

PATE RR, PRATT M, BLAIR SN, HASKELL WL, MACERA CA, BOUCHARD C, et al. Physical activity and public health. A recommendation from the Centers for Disease Control and Prevention and the American College of Sports Medicine. JAMA. v. 273, n. 5, p. 402-407, 1995. 
PINHEIRO, T. M. M. Saúde do Trabalhador Rural: RENAST: condições de vida, trabalho, saúde e doença dos trabalhadores rurais no Brasil. Disponível em <www.medicina.ufmg.br/dmps/2006/saude_trabalhador_rural.pdf>. Acesso em: 10 maio 2009.

PITANGA, F. J.; LESSA, I. Prevalence and variables associated with leisure-time sedentary lifestyle in adults. Cadernos de Saúde Pública, v. 21, n. 3, p. 870-877, 2005.

ROSA, A. A.; GONÇALVES S.C.; STEFANI S.D.; MARTINS S. O.; ROSA D. D.; HUNSCHE A., AZEREDO R. M.; SCHÖNELL L. H.; RUSCHEL M. M.; DE BARROS S. G. S. Percepção e registro de abuso de álcool e de doenças relacionadas num hospital geral universitário. Revista da Associação Médica Brasileira, v. 44, n. 4, p. 335-339, 1998.

ROSEMBERG, J. Nicotina: droga universal. São Paulo: Secretaria de Estado da Saúde SES/CVE; 2003. Disponível em: <www.inca.gov.br/tabagismo/publicacoes/nicotina.pdf>. Acesso em: 10 maio 2009.

SALLES-COSTA, R.; HEILBORN, M.L.; WERNECK, G.L.; FAERSTEIN, E.; LOPES. C. S. Gênero e prática de atividade física no lazer. Cadernos de Saúde Pública, v. 19, n. 2, p. 325-333, 2003.

ALMEIDA-FILHO, N. SANTANA, V. S.; MARI, J. . Bases Hisóricas de Epidemiológia Psiquiátrica. Revista da ABP-APAL, v. 9, n. 3, p. 107-112, 1987.

SANTOS, J. F. S.; COELHO, C. W. Atividade física e obesidade em trabalhadores da indústria. Revista Lecturas, Educación Física y Deportes, v. 9, n. 67, 2003.

SÁVIO, K. E. O.; et al. Sexo, renda e escolaridade associados ao nível de atividade física de trabalhadores. Revista de Saúde Pública, v. 42, n. 3, p. 457-463, 2008.

TRIOLA, M.F. Introdução à estatística. 9. ed. Rio de Janeiro: LTC, 2005.

WILLIAMS, P. Physical fitness and activity as separate heart disease risk factors: a meta-analysis. Medicine \& Science in Sports \& Exercise, v. 33, n.5, p. 754-761, 2001.

WORLD BANK. Tobacco \& Health in the Developing World. A Background Paper for the High Level Round Table On Tobacco Control and Development Policy. Organized by the European Commission in collaboration with the World Health Organization and the World Bank, Brussels, 2003.

WORLD HEALTH ORGANIZATION. Global strategy on diet, physical activity and health. Geneva, 2004.

WORLD HEALTH ORGANIZATION. Diet, nutrition and prevention of chronic diseases. Report of the joint WHO/FAO expert consultation, Geneva, 2003. 